\title{
Fortbildung
}

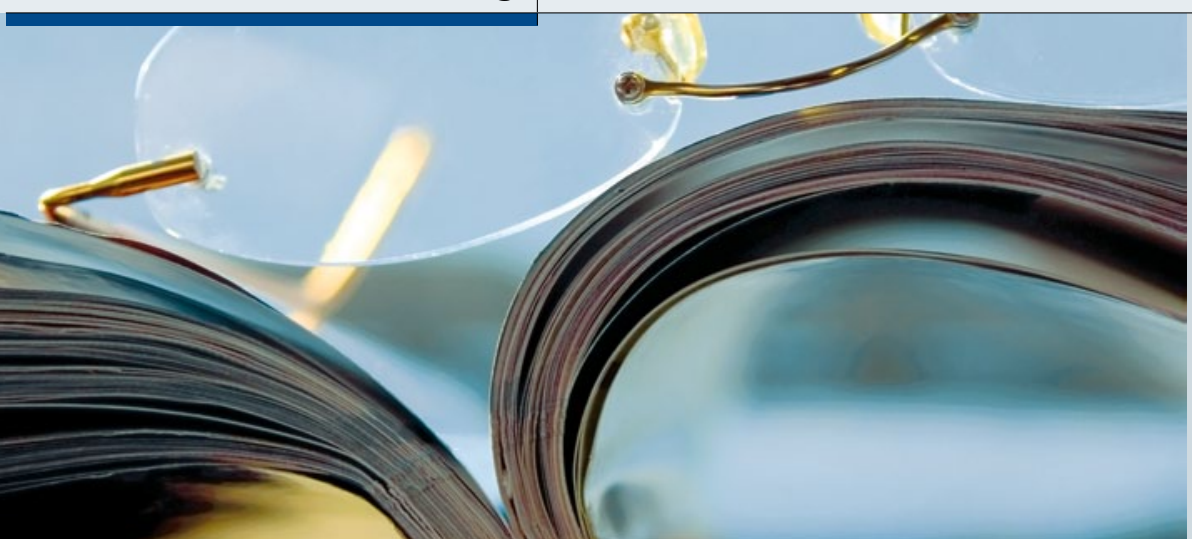

In der Rubrik "Literatur kompakt" werden wichtige Arbeiten aus der internationalen Fachliteratur referiert und auf den Punkt gebracht.

\section{Leitliniengerechte Therapie der Fibromyalgie}

Innerhalb der deutschen interdisziplinären Vereinigung für Schmerztherapie hat sich eine Arbeitsgruppe gebildet, die sich mit der Diagnostik und Therapie der Fibromyalgie beschäftigt. Diese Arbeitsgruppe hat jüngst die S3-Leitlinie Fibromyalgie überarbeitet. Da dieses Krankheitsbild oft überdiagnostiziert wird und sowohl fälschlich vollständig ignoriert als auch fälschlich dramatisiert wird, ist eine gute Analyse der gesamten wissenschaftlichen Literatur hilfreich.

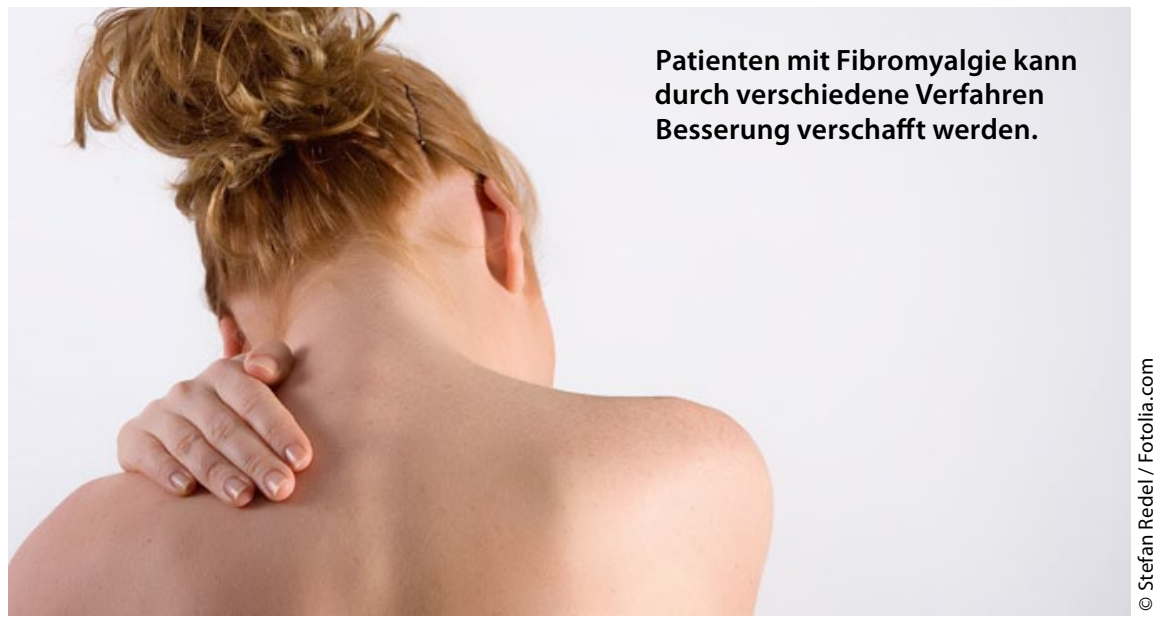

nsbesondere wurden in drei Publikationen die verschiedenen Therapieverfahren systematisch analysiert $[1,2,3]$. Es stellte sich heraus, dass Amitriptylin und Duloxetin in der medikamentösen Therapie der Fibromyalgie die einzigen wirksamen Medikamente sind. Für Duloxetin gilt dies nur in Kombination mit komorbiden Depressionen oder Angststörungen. Pregabalin scheint ebenfalls effektiv zu sein, dies hat jedoch nur eine schwache Evidenz. Opioide sind nicht wirksam. Zudem wurden psychotherapeutische Verfahren einer Metaanalyse unterzogen. Hier zeigte sich, dass die kognitive Verhaltenstherapie in Verbindung mit aerobischen Übungsbehandlungen einen eindeutigen signifikanten Effekt auf die Fib- romyalgie hat. Eine Entspannungstherapie (z.B. progressive Muskelrelaxation oder autogenes Training) als alleinige Therapie ist dagegen nicht hilfreich.

Im Bereich der physiotherapeutischen und physikalischen Therapie wurde deutlich, dass eine leichte bis mäßige Intensität von aerobischen Übungsbehandlungen und muskuläres Stärkungstraining eine Evidenz in der Behandlung der Fibromyalgie hat. Die Fülle von weiteren physikalischen Therapieverfahren konnte sämtlich keine seriöse Evidenz zeigen. Nicht empfohlen werden von der Arbeitsgruppe die chiropraktische Therapie, Lasertherapie, Magnetfeldtherapie, Massagen und die transkranielle Gleichstromstimulation.
Kommentar: Es ist mehr als lobenswert und hilfreich, dass die wissenschaftliche Literatur zum Thema Fibromyalgie durch die Arbeitsgruppe genau analysiert und zusammengefasst worden ist. Die Ergebnisse werden klar dargestellt und bieten inzwischen jedem schmerztherapeutisch Tätigen eine Leitlinie, mit welchen therapeutischen Verfahren Fibromyalgie behandelt werden kann. Auf der einen Seite zeigt sich, dass ein therapeutischer Nihilismus nicht angezeigt ist, denn es gibt eine Reihe von Verfahren, die evidenzbasiert wirksam sind, sowohl medikamentös als auch psycho- und physiotherapeutisch. Auf der anderen Seite gibt es zahlreiche Verfahren, die selbst von Fachgesellschaften empfohlen werden und keinerlei seriöse wissenschaftliche Evidenz aufweisen. Insofern bleibt natürlich noch Forschungsbedarf in der Behandlung dieser Erkrankung, zumal die Therapieeffekte nicht immer befriedigend sind.

Prof.Dr.med. Stefan Evers

1. Sommer C, Häuser W, Alten R, Petzke F, Späth $M$, Tölle T, Üçeyler N, Winkelmann A, Winter E, Bär KJ. Medikamentöse Therapie des Fibromyalgiesyndroms. Systematische Übersicht und Metaanalyse. Schmerz 2012; 26: 297-310

2. Köller V, Häuser W, Klimczyk K, Kühn-Becker H, Settan M, Weigl M, Bernardy K. Psychotherapie von Patienten mit Fibromyalgiesyndrom. Systematische Übersicht, Metaanalyse und Leitlinie. Schmerz 2012; 26: 291-6

3. Winkelmann A, Häuser W, Friedel E, MoogEgan M, Seeger D, Settan M, Weiss T, Schiltenwolf $M$. Physiotherapie und physikalische Verfahren beim Fibromyalgiesyndrom

4. Systematische Übersicht, Metaanalyse und Leitlinie. Schmerz 2012; 26: 276-86

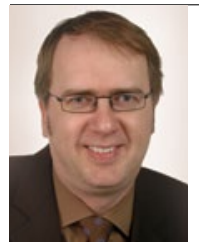

Prof. Dr. med. Dr. phil. Stefan Evers Klinik und Poliklinik für Neurologie Universitätsklinikum Münster

Albert-Schweitzer-Str. 33, 48129 Münster 